\title{
Review of: "Neutralization of SARS-CoV-2 spike 69/70 deletion, E484K, and N501Y variants by BNT162b2 vaccine- elicited sera"
}

Helene Banoun

Potential competing interests: The author(s) declared that no potential competing interests exist.

Neutralization of SARS-CoV-2 spike 69/70 deletion, E484K and N501Y variants by BNT162b2 vaccine-elicited sera Xie, X., Liu, Y., Liu, J. et al. Neutralization of SARS-CoV-2 spike 69/70 deletion, E484K and N501Y variants by BNT162b2 vaccine-elicited sera. Nat Med 27, 620-621 (2021). https://doi.org/10.1038/s41591-021-01270-4

This publication by Pfizer employees attempts to show that the antibodies of people vaccinated with Pfizer's anti-Covid-19 vaccine are effective against the UK (B.1.1.7) and SA (B.1.351) variants of SARS-CoV-2.

They produce 3 pseudoviruses expressing a spike protein containing the characteristic mutations of these variants.

These viruses express either the mutations:

N501Y, SA and UK in the RBD of the spike which allows the virus to infect the mouse,

or the 69/70 N501 deletion and the D614 mutation of the UK

or the following 3 mutations of the SA variant: E484K+ N501+ D614.

The neutralizing antibody titers are measured in the sera of 20 vaccinated persons.

Here are the results: The geometric mean neutralization titers (GMTs) of 20 human sera induced by the BTN162b2 vaccine against the three mutant viruses were 0.81 to 1.46 times higher than the GMTs against the parental virus, indicating small effects of these mutations on neutralization by sera induced by two doses of BNT162b2.

\section{Reminder}

Compared to line B.1, B.1.1.7 has 19 major mutations, including 8 on protein S.

Among them:

N501Y which, by slightly modifying the end of the S protein, seems to improve the binding between this protein and the ACE2 receptor of the target cells;

$\mathrm{P} 681 \mathrm{H}$ which seems to increase the production of $\mathrm{S}$ protein by infected cells two deletions (H69-V70 and Y144/145) which could modify the three-dimensional structure of the S protein. Compared to line B.1, B.1.351 has 13 major mutations, including 8 on protein S.

These include:

N501Y and K417N which appear to improve the binding between the S protein and the ACE2 receptor of target cells; E484K which, by modifying the shape of the end of $S$, could allow the virus to partially escape antibodies resulting from an 
infection or vaccination using the S protein.

This publication is criticizable for the following reasons:

1) Why not have tested the antibodies of the vaccinated on pseudoviruses expressing all the mutations of the variants?

Indeed, other studies show that it is important to test the resistance of the variants to the antibodies on pseudoviruses possessing all the mutations and deletions of the spike protein and not only those of the RBD: the pseudoviruses possessing only a part of the mutations are less neutralized than those expressing them all. ${ }^{\text {ii }}$

This is related to the fact that the in vivo fitness of the virus depends on the set of mutations characteristic of a clade and not on an isolated mutation. iii

2) Moreover, most publications use pseudoviruses whereas live viruses have been shown to show lower neutralization (Mahdi et al, 2020, note ii) : although this late study concerns the efficacy of the Astra Zeneca vaccine (ChAdOx1 nCoV-19 Covid-19), it is likely that the same phenomenon occurs with mRNA vaccines.

3) In spite of these probable biases, other publications show the reduction of the neutralization of pseudoviruses by the antibodies of people vaccinated with the Pfizer vaccine:

3a Wang P, Liu L, Iketani S, et al. Increased resistance of SARS-CoV-2 variants B.1.351 and B.1.1.7 to antibody neutralization. January $26,2021^{\mathrm{iv}}$.

They use here a VSV-based SARS- CoV-2 pseudoviruses that contain each of the individual mutations as well as one with all 8 mutations of the B.1.1.7 variant (UK $\triangle 8)$ and another with all 9 mutations of the B.1.351 variant (SA $\triangle 9$ ). The resistance of B.1.351 is largely conferred by $242-244$ del and/or R246l.

\section{Vaccine sera}

Each vaccinee serum sample was assayed for neutralization against B.1.1.7, B.1.351, and WT viruses. Neutralizing activity against B.1.1.7 was essentially unchanged, but significantly lower against B.1.351 (12.4 fold, Moderna; 10.3 fold, Pfizer). No single mutation in B.1.1.7 has an appreciable impact on the neutralizing activity of vaccinee sera. The loss of neutralizing activity against $\mathrm{SA} \triangle 9$ is largely consistent with the loss in B.1.351 live virus neutralization.

3b Another publication by Pfizer and BioNTech employees[V]

This paper proves the opposite of what is written in the abstract, the Pfizer vaccine loses a lot of efficacy in vitro against the new variants.

The reference strain used does not contain the D614G mutation contrary to the other studies In addition, the data clearly show a downward trend in almost all sera. 
The article states in the abstract that "the immunized sera had equivalent neutralizing titers for both variants." Again, this is not correct. Twelve out of sixteen individuals had a titer ratio of less than one when comparing the activity of B.1.1.7 to that of the Wuhan strain. A conservative a-parametric test (coin flip) shows that the reduction is statistically significant $(p<0.05)$. It is incorrect to say that they have "equivalent" titers.

3c mRNA vaccine-elicited antibodies to SARS-CoV-2 and circulating variants[vi]

As in the study criticized here, the authors use pseudoviruses each expressing only either one of the characteristic mutations of the UK or SA variants, or a combination of some. Yet they show the reduction of neutralization by Pfizer (or Moderna) vaccine sera and the near disappearance of neutralization for the majority of monoclonal antibodies derived from vaccine sera.

A 3-fold reduction is found for the combination K417N:E484K:N501Y

They conclude that the plasma neutralizing activity elicited by mRNA vaccination is variably but significantly less effective against particular RBD mutants in the tested panel.

It is also possible that with the gradual disappearance of vaccine immunity, the efficacy of these vaccines erodes faster against the variants over time.

3d Neutralization of viruses with European, South African, and United States SARS-CoV-2 variant spike proteins by convalescent sera and BNT162b2 mRNA vaccine-elicited antibodies [vii]

The authors of this study work on pseudoviruses expressing the full mutant spike protein of the variants. Their results suggest that antibodies elicited by primary infection and by the BNT162b2 mRNA vaccine are likely to maintain protective efficacy against B.1.1.7 and most other variants but that the partial resistance of virus with the B.1.351 spike protein could render some individuals less well protected, supporting a rationale for the development of modified vaccines containing E484K.

Vaccine elicited antibodies neutralized virus with the B.1.1.7 spike protein with titers similar to D614G virus and neutralized virus with the B.1.351 spike with, on average, a 3-fold reduction in titer The reduction in titer was attributable to the E484K mutation in the RBD.

The partial resistance of B.1.351 to vaccine elicited antibody could result in reduced level of protection in individuals with below average neutralizing titer while B.1.351 and E484K were neutralized with a 3.1- and 4.3-fold decrease in titer, especially since the vaccinated have a zero level of neutralizing antibodies between 0 and 7 days after the vaccine: they could be even more sensitive to the variants during this period.

3e Another study[viii] by Moderna employees on the neutralization of pseudoviruses by the antibodies of Moderna vaccinees shows that the reduction in neutralization of pseudoviruses expressing a spike with the K417N-E484K-N501YD614G mutations is 2.7 times, whereas it is 6.4 times when the expressed spike has all the B.1.351 variant mutations. Therefore, this is not the Pfizer vaccine but the other mRNA vaccine with identical function. 


\section{4) Are the plasmids expressed correctly?}

Moreover, according to Tada et al.[ix] the plasmids incorporated in some pseudoviruses can express the spike protein very poorly, especially for pseudoviruses comprising the protein with key mutations of the mutants: it should have been verified in the study under criticism that the mutations studied do not disrupt the expression of the protein and this for each mutation and for the associated mutations (quote from Tada et al. : Analysis of the B.1 .1.7 and B.1.351 spike proteins containing the full complement of mutations showed that the B.1.351 spike protein was stable and processed as wild-type while the B.1.1.7 was poorly expressed and little of the protein was present on virions)

\section{5) All this is far from what is happening in the real world with vaccinated individuals:}

A study[x], conducted in Israel on people vaccinated and not vaccinated with Pfizer shows (according to the reviewers of the study[xi]) that partially vaccinated individuals were more likely than unvaccinated individuals to be infected with the B.1.1.7 strain ("U.K. variant") than with the wildtype strain. Fully vaccinated individuals were more likely than unvaccinated individuals to be infected with the B.1.351 strain ("South African variant") than with the wildtype strain.

These results, if verified, suggest that the B.1.1.7/U.K. variant is more effective at breaking through partial vaccination than the wildtype strain; and that the B.1.351/S.A. variant is more effective at breaking through full vaccination than the wildtype strain.

(i)

https://assets.publishing.service.gov.uk/government/uploads/system/uploads/attachment data/file/961299/Variants of Co ncern VOC Technical Briefing 6 England-1.pdf

(ii )https://www.nejm.org/doi/full/10.1056/NEJMoa2102214 Madhi SA, Baillie V, Cutland CL, Voysey M, Koen AL, Fairlie L, Padayachee SD, Dheda K, Barnabas SL, Bhorat QE, Briner C, Kwatra G, Ahmed K, Aley P, Bhikha S, Bhiman JN, Bhorat AE, du Plessis J, Esmail A, Groenewald M, Horne E, Hwa SH, Jose A, Lambe T, Laubscher M Malahleha M, Masenya M, Masilela M, McKenzie S, Molapo K, Moultrie A, Oelofse S, Patel F, Pillay S, Rhead S, Rodel H, Rossouw L, Taoushanis C, Tegally H, Thombrayil A, van Eck S, Wibmer CK, Durham NM, Kelly EJ, Villafana TL, Gilbert S, Pollard AJ, de Oliveira T, Moore PL, Sigal A, Izu A; NGS-SA Group Wits-VIDA COVID Group. Efficacy of the ChAdOx1 nCoV-19 Covid-19 Vaccine against the B.1.351 Variant. N Engl J Med. 2021 Mar 16:NEJMoa2102214. doi: 10.1056/NEJMoa2102214. Epub ahead of print. PMID: 33725432; PMCID: PMC7993410.

(iii) Evolution of SARS-CoV-2: review of mutations, role of the host immune system, Banoun H, Nephron (2020, in press) DOI: $10.1159 / 000515417$ 
(iv) (https://www.biorxiv.org/content/10.1101/2021.01.25.428137v1

B.1.351 is markedly more resistant to neutralization by convalescent plasma ( 9.4 fold) and vaccinee sera (10.3-12.4 fold)

(V) Muik A, Wallisch A-K, Sänger B, et al. Neutralization of SARS-CoV-2 lineage B.1.1.7 pseudovirus by BNT162b2 vaccine-elicited human sera. Science 2021 January 29 (Epub ahead of print).

Alexander Muik, Ann-Kathrin Wallisch, Bianca Sänger, Kena A. Swanson, Julia Mühl, Wei Chen, Hui Cai, Ritu Sarkar, Özlem Türeci, Philip R. Dormitzer, Ugur Sahin

bioRxiv 2021.01.18.426984; doi: https://doi.org/10.1101/2021.01.18.426984

(VI) Wang, Z., Schmidt, F., Weisblum, Y. et al. mRNA vaccine-elicited antibodies to SARS-CoV-2 and circulating variants. Nature (2021). https://doi.org/10.1038/s41586-021-03324-6

[VII] Tada T, Dcosta BM, Samanovic-Golden M, Herati RS, Cornelius A, Mulligan MJ, Landau NR. Neutralization of viruses with European, South African, and United States SARS-CoV-2 variant spike proteins by convalescent sera and BNT162b2 mRNA vaccine-elicited antibodies. bioRxiv [Preprint]. 2021 Feb 7:2021.02.05.430003. doi: 10.1101/2021.02.05.430003. PMID: 33564768; PMCID: PMC7872356. https://pubmed.ncbi.nlm.nih.gov/33564768/

[VIII) https://www.biorxiv.org/content/10.1101/2021.01.25.427948v1 mRNA-1273 vaccine induces neutralizing antibodies against spike mutants from global SARS-CoV-2 variants Kai Wu, Anne P. Werner, Juan I. Moliva, Matthew Koch, Angela Choi, Guillaume B. E.Stewart-Jones, Hamilton Bennett, Seyhan Boyoglu-Barnum, Wei Shi, Barney S.Graham, Andrea Carfi, Kizzmekia S. Corbett, Robert A. Seder, Darin K. Edwards

bioRxiv 2021.01.25.427948; doi: https://doi.org/10.1101/2021.01.25.427948

[IX] Tada T, Dcosta BM, Samanovic-Golden M, Herati RS, Cornelius A, Mulligan MJ, Landau NR. Neutralization of viruses with European, South African, and United States SARS-CoV-2 variant spike proteins by convalescent sera and BNT162b2 mRNA vaccine-elicited antibodies. bioRxiv [Preprint]. 2021 Feb 7:2021.02.05.430003. doi: 10.1101/2021.02.05.430003. PMID: 33564768; PMCID: PMC7872356. https://pubmed.ncbi.nlm.nih.gov/33564768/

$[X]$ Evidence for increased breakthrough rates of SARS-CoV-2 variants of concern in BNT162b2 mRNA vaccinated individuals

Talia Kustin, Noam Harel, Uriah Finkel, Shay Perchik, Sheri Harari, Maayan Tahor, Itamar Caspi, Rachel Levy, Michael Leschinsky, Shifra Ken Dror, Galit Bergerzon, HalaGadban, Faten Gadban, Eti Eliassian, Orit Shimron, Loulou Saleh, Haim Ben-Zvi, Doron Amichay, Anat Ben-Dor, Dana Sagas, Merav Strauss, Yonat Shemer Avni, AmitHuppert, Eldad Kepten, Ran D. Balicer, Doron Nezer, Shay Ben-Shachar, Adi Stern medRxiv 2021.04.06.21254882; doi:https://doi.org/10.1101/2021.04.06.21254882 
[XI] https://rapidreviewscovid19.mitpress.mit.edu/pub/fyz79592/release/5 